\title{
5.6 Aortic Pressure Behind Flow Disorganization in Aneurismal Aorta: A Magnetic Resonance Imaging Study
} \author{
Elie Mousseaux ${ }^{2}$, Nadjia Kachenoura ${ }^{1}$ \\ ${ }^{1}$ Sorbonne Université, INSERM, CNRS, Laboratoire d'Imagerie Biomé-dicale, Paris, France \\ ${ }^{2}$ Hôpital Européen Georges Pompidou, INSERM 970, Paris, France \\ ${ }^{3}$ CMLA, ENS Cachan, CNRS, Université Paris-Saclay, 94235 Cachan, France \\ ${ }^{4}$ LIMSI, CNRS, Université Paris-Saclay, Orsay, France
}

Kevin Bouaou ${ }^{1,}$, Thomas Dietenbeck ${ }^{1}$, Gilles Soulat ${ }^{2}$, Sophia Houriez-Gombaud-Saintonge ${ }^{1}$, Ioannis Bargiotas ${ }^{3}$, De Cesare Alain ${ }^{1}$, Umit Gencer ${ }^{2}$, Alain Giron ${ }^{1}$, Alban Redheuil ${ }^{1}$, Emilie Bollache ${ }^{1}$, Didier Lucor ${ }^{4}$,

\section{ABSTRACT}

Purpose: Understanding and prediction of ascending thoracic aortic aneurysms (ATAA) progression are not well established yet and aortic dissection is frequently occurring on normally sized and mildly dilated aortas. Despite known theoretical associations between pressures and blood flow patterns there are no studies focusing on their simultaneous evaluation. Our aim was to propose a comprehensive and quantitative evaluation of pressure-flow-wall interplay from $4 \mathrm{D}$ flow MRI in the setting of aortic dilation.

Methods: We studied 12 patients with ATAA ( $67 \pm 14$ years, 7 males) and 12 healthy subjects $(63 \pm 12$ years, 8 males) who underwent $4 \mathrm{D}$ flow MRI. The segmented velocity fields were used to estimate: 1) local ascending aorta (AA) pressure changes from Navier-Stokes-derived relative pressure maps (AADP, $\mathrm{mmHg}$ ), 2) AA wall shear stress (AAWSS, Pa) by estimating local velocity derivatives at the aortic borders, 3 ) aortic flow vorticity using the $\lambda 2$ method (AAV, s-1).

Results: AA local pressure change (AADP) was significantly associated with both AAV $(r=0.55, p=0.006)$ and AAWSS $(r=0.69, p<0.001)$ and both associations remained significant after adjustment for diameter, age and BSA $(p=0.007$ and $p=0.003$ respectively). Such positive associations indicate that local pressure variations affect local blood flow, generating flow current from high to low pressures and subsequently vortices with the underlying stress exerted on the AA wall.

Conclusion: Local variations in aortic pressures, measured using 4D flow MRI, are associated with flow disorganization as quantified by vorticity and with the increase in the stress exerted on the aortic wall, as quantified by wall shear stress.

(c) 2019 Association for Research into Arterial Structure and Physiology. Publishing services by Atlantis Press International B.V. This is an open access article distributed under the CC BY-NC 4.0 license (http://creativecommons.org/licenses/by-nc/4.0/). 